\title{
Skin oxygenation impairment is associated with increased total cholesterol level in children with short-lasting type 1 diabetes mellitus
}

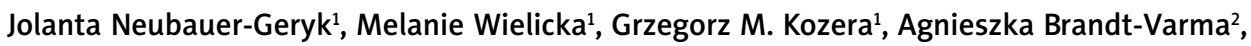 \\ Anna Wołoszyn-Durkiewicz², Małgorzata Myśliwiec ${ }^{2}$, Leszek Bieniaszewski ${ }^{1}$ \\ ${ }^{1}$ Clinical Physiology Unit, Medical Simulation Centre, Medical University of Gdansk, Gdansk, Poland \\ 2Department of Paediatrics, Diabetology and Endocrinology, Medical University of Gdansk, Gdansk, Poland \\ Adv Dermatol Allergol 2021; XXXVIII (4): 615-621 \\ DOI: https://doi.org/10.5114/ada.2020.98220
}

\begin{abstract}
Introduction: Transcutaneous oxygen pressure $(\mathrm{tcPO})$ is a non-invasive method of measuring skin oxygenation that may reflect its superficial perfusion. Skin microvasculature may be impaired in patients with late onset of type 1 diabetes (DM1). However, its condition in children has not been fully determined.

Aim: To compare tcPO in children with short-lasting non-complicated DM1 and age-matched healthy controls with regard to concomitant vascular risk factors.

Material and methods: The study group consisted of 51 paediatric patients aged 14.9 (8.4-18.0) years with shortlasting DM1 without clinical evidence of diabetic micro- or macroangiopathy and 28 control subjects aged 14.8 (11.3-17.7) years. TcPO, was tested prior, during and after applying post-occlusive reactive hyperaemia (PORH) test in standardized conditions. Biochemical parameters were assessed and then compared between the groups.

Results: $\mathrm{TCPO}_{2}$ at maximal ischemia during PORH was higher in the DM1 patients than in healthy controls (2.4 (0.7-18.8) vs. $1.6(0.4-12.0), p=0.002)$. No differences were found regarding the $\mathrm{tcPO}_{2}$ measurements recorded prior to ischemia or after recovery. In DM1, concentrations of total cholesterol, triglycerides, $\mathrm{HbA}_{1 c}$ and TSH were significantly higher than in healthy controls. The fT4 levels were significantly lower in the DM1 group. After adjusting for lipid levels, no differences in $\mathrm{tcPO}_{2}$ were found, and a multivariate analysis showed the cholesterol levels have a significant impact on $\mathrm{tcPO}$, response to maximal ischemia.

Conclusions: Our results indicate that increased lipid levels are responsible for the impaired skin response to ischemic stimuli in short-lasting DM1. This supports the importance of aggressive lipid control in prevention of early onset microangiopathy in those patients.
\end{abstract}

Key words: diabetes mellitus, children, skin microangiopathy, transcutaneous oxygen pressure, post occlusive reactive hyperaemia, lipids.

\section{Introduction}

Patients with type 1 diabetes are at high risk for early skin microcirculatory impairment [1-3]. Its functional impairment, which precedes structural pathology, may be detected with the use of venous occlusion (VO) or post-occlusive reactive hyperaemia (PORH) tests, both of which involve shear stress and its consequences [4-7]. Microvascular function using capillary recruitment during $\mathrm{PORH}$ is secondary to ischaemia and related to endothelium-dependent vasodilatation at the level of precapillaries mediated by an axonal reflex and the endothelium derived hyperpolarizing factor [8, 9].
The influence of these provocation tests on tissue oxygenation may be assessed with transcutaneous oxygen pressure $\left(\mathrm{tcPO}_{2}\right)$ measurements, which also allow for simultaneous functional evaluation of skin microcirculation. In recent years, the practical value of $\mathrm{tc} \mathrm{PO}_{2}$ in screening and follow up of overt vascular disease has been well documented [10-16]. TCPO, values have been also confirmed as an independent prognostic marker for 1-year mortality among patients with type 1 diabetes and diabetic foot ulcers [17]. However, despite its high diagnostic value, tcPO $\mathrm{P}_{2}$ measurements have not been recently reported in younger populations with less advanced skin

Address for correspondence: Jolanta Neubauer-Geryk MD, PhD, Clinical Physiology Unit, Medical Simulation Centre, Medical University of Gdansk, 25 Dębowa St, 80-204 Gdansk, Poland, phone: +48 58349 11 89, e-mail: jolaneub@gumed.edu.pl Received: 4.02.2020, accepted: 16.03.2020. 
microangiopathy. The influence of lipid and thyroid hormone levels on $\mathrm{tcPO}_{2}$ also remains unclear.

\section{Aim}

Our aim was to compare tcPO in children with shortlasting non-complicated type 1 diabetes mellitus (DM1) and age-matched healthy controls with regard to serum lipid and thyroid hormone concentrations.

\section{Material and methods}

The study group consisted of two subgroups: 51 paediatric patients with type 1 diabetes (median duration: 4.7 (1.2-9.9) years, median age at onset: 10.4 (2.1-13.9) years, and 28 age-matched control subjects (Table 1). Patients were recruited from the Department of Paediatrics, Diabetology and Endocrinology at Medical University of Gdansk in 2014-2018. Only patients with short-lasting (disease duration of less than 10 years) diabetes without clinical evidence of micro- or macroangiopathic complications of type 1 diabetes were included in the study. The only medication the patients were using was insulin, either through a pump or a pen. Based on medical history, physical examination and biochemical analysis, none of the study subjects had any form of microangiopathy, including retinopathy, nephropathy or neuropathy. All examinations were performed between 8:00 A.M. and 1:00 P.M. The study protocol included medical history, $\mathrm{tcPO}_{2}$ and laboratory testing. The lack of microangiopathy (reti- nopathy, nephropathy and neuropathy) was confirmed using previously published criteria [18-20]. The patients from all study groups were euthyroid at the time of investigation.

All procedures performed in the studies involving human participants were in accordance with the ethical standards of the Medical Ethics Committee of the Medical University of Gdansk, Poland and with the 1964 Helsinki declaration and its later amendments or comparable ethical standards. The study protocol was approved by the Medical Ethics Committee of the Medical University of Gdansk (NKBBN/277/2014; NKBBN/277-512/2016). Upon entering the study each participant has given informed consent. Parents agreed to carry out microcirculation tests and they were present during the tests.

\section{Transcutaneous oxygen pressure examination}

During the examination, patients remained in a comfortable sitting position with the upper limb freely supported at the heart level. Core body temperature was controlled with the use of a contactless thermometer (Novama - Model NT 19) and was within normal range in all examined patients and control subjects. The room temperature was controlled by air conditioning and was kept the same during all tests.

Transcutaneous oxygen pressure examination was assessed with PeriFlux System 5000 (Perimed AB, Sweden). The level of $\mathrm{tcPO}_{2}$ is based on the amount of oxygen that diffuses from the capillaries through the epidermis to the electrode and thus provides information

Table 1. Characteristics of study groups

\begin{tabular}{|c|c|c|c|}
\hline Characteristics & $\begin{array}{l}\text { Healthy controls } \\
\qquad n=28\end{array}$ & $\begin{array}{c}\text { Diabetic patients } \\
\quad n=51\end{array}$ & $P$-value \\
\hline Male gender (\%) & 54 & 43 & 0.37 \\
\hline Body mass [kg] & $53.4(29.0-80.5)$ & $57.4(28.8-95.7)$ & 0.27 \\
\hline Body mass index $\left[\mathrm{kg} / \mathrm{m}^{2}\right]$ & $19.3(14.4-24.7)$ & $20.5(14.5-29.7)$ & 0.46 \\
\hline Age [years] & $14.8(11.3-17.7)$ & $14.9(8.4-18.0)$ & 0.39 \\
\hline Age at onset of type 1 diabetes [years] & - & $10.4(2.1-13.9)$ & - \\
\hline Duration of DM [years] & - & $4.7(1.2-9.9)$ & - \\
\hline Insulin dose [units/day] & - & $44(21-75)$ & - \\
\hline Treatment with pump (\%) & - & 47 & - \\
\hline $\mathrm{HbA}_{1 \mathrm{c}}(\%) /[\mathrm{mmol} / \mathrm{mol}]$ & $\begin{array}{c}5.3 / 5.9 \\
(4.8-5.6) /(5.1-6.3)\end{array}$ & $\begin{array}{c}7.6 / 9.5 \\
(5.3-13.6) /(5.9-19.1)\end{array}$ & $<0.001$ \\
\hline $\mathrm{TSH}[\mathrm{mIU} / \mathrm{l}]$ & $1.2(0.6-2.4)$ & $1.7(0.6-3.6)$ & 0.001 \\
\hline fT4 [pmol/l] & $13.6(11.4-16.8)$ & $12.3(9-14.9)$ & $<0.001$ \\
\hline Total cholesterol [mg/dl] & $163(120-218)$ & $177(119-288)$ & 0.005 \\
\hline Triglycerides [mg/dl] & $54(36-117)$ & $70(37-294)$ & 0.007 \\
\hline Cholesterol HDL [mg/dl] & $51(41-82)$ & $55(33-120)$ & 0.22 \\
\hline Cholesterol LDL [mg/dl] & $95(64-127)$ & $102(61-188)$ & 0.13 \\
\hline
\end{tabular}

values are presented as median and range. 


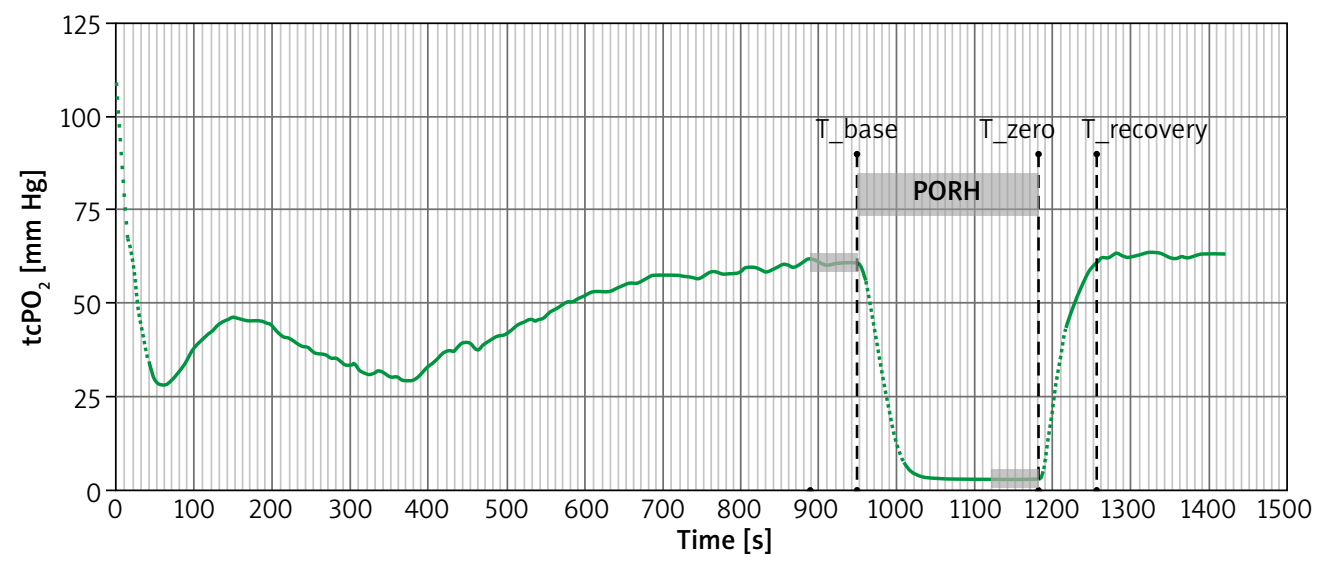

Figure 1. The scheme of $\mathrm{tcPO}_{2}$ measurement during the post-occlusive reactive hyperaemia (PORH) test

about the body's ability to deliver oxygen to tissues [18]. In the $\mathrm{tcPO}_{2}$ measurement, sensors that contain a pair of polarized electrodes are used, enabling the determination of oxygen content in a given volume. The classic oxygen electrode contains a silver chloride anode and a cathode made of gold or platinum, separated with liquid electrolytes. Electrodes are bound by a polymer membrane (Teflon, polyethylene or silicone polymer), which selectively permeates oxygen from the examined area of the skin [21]. The output current is proportional to the partial pressure of oxygen in tissues [22] and skin circulation, oxyhemoglobin dissociation and tissue metabolic activity. An electrochemical electrode and a local heating system were used for the measurements. In Periflux System 5000 it is possible to heat up the electrode to a temperature range of $37-45^{\circ} \mathrm{C}$. The skin temperature of $43^{\circ} \mathrm{C}$ was chosen for the purpose of achieving local vascular congestion. A comparable temperature was used in a study by de Meijer [23] and was proposed in "The tcPO handbook" [24].

After calibrating the device and heating the Clarketype electrode to $43^{\circ} \mathrm{C}$, the electrode was placed in the adhesive groove on the central part of the hairless forearm. During the PORH test, the blood pressure cuff was placed around the patient's arm and inflated to a pressure $50 \mathrm{~mm} \mathrm{Hg}$ greater than systolic blood pressure for 4 min [25]. $\mathrm{TCPO}_{2}$ was continuously recorded during rest (baseline), occlusion (PORH) and after cuff release (recovery period) (Figure 1).

The curve analysis included identification of specific time points i.e. T_base as the moment of initiation of occlusion, T_zero as the moment of end of occlusion and T_recovery as the moment when pre-occlusion value was observed again.

The following parameters were determined:

- $\mathrm{tcPO}_{2-}$ base - the mean value of $\mathrm{tcPO}_{2}$ within $60 \mathrm{~s}$ before T_base;
- $\mathrm{tcPO}_{2}$ zero - the mean value of $\mathrm{tcPO}_{2}$ within $60 \mathrm{~s}$ before the T_zero;

- Slope - it was determined as the ratio of $\mathrm{tcPO}_{2}$ diff and TTR, where:

- $\mathrm{tcPO}_{2-}$ diff - the difference between $\mathrm{TcPO}_{2}$ base and tcPO_zero,

- TTR - time to reach baseline value after occlusion (T_recovery - T_zero).

\section{Statistical analysis}

All the analyses were performed using Statistica data analysis software system, version 12 (StatSoft, Inc., Tulsa, OK, USA). Shapiro-Wilk tests were performed to analyse the distribution of continuous variables. Values are expressed either as median and range or as mean and standard deviation, as appropriate. For group's changes, Wilcoxon test was used. The comparison between groups was performed using the Mann-Whitney test. The $\chi^{2}$ test was used to compare the proportion of genders and the frequency of mild and severe hypoglycaemia. The comparison between groups with regard to associated continuous variables was performed using the General Linear Model with Fisher test for post-hoc analysis when needed, multivariate analyses were performed to assess the independent impact of biochemical parameters on skin oxygenation. The value of $p<0.05$ was deemed statistically significant.

\section{Results}

Transcutaneous oxygen pressure at maximal ischemia ( $\mathrm{tcPO}_{2}$ zero) was higher in children with type 1 diabetes than in healthy controls $(p=0.002)$. There were no differences between groups regarding tc $\mathrm{PO}_{2}$ prior to $\mathrm{PORH}$ initiation ( $\mathrm{tcPO} \mathrm{P}_{2-}$ base) or tc $\mathrm{PO}_{2}$ at recovery after $\mathrm{PORH}$ (tcPO $\mathrm{C}_{2}$ diff and TTR) (Table 2). Serum concentrations of total cholesterol, triglycerides, $\mathrm{HbA}_{1 \mathrm{c}}$ and TSH were higher, and fT4 were lower in children with type 1 
Table 2. Characteristics of $\mathrm{tcPO}_{2}$ parameters in studied groups

\begin{tabular}{|c|c|c|c|}
\hline Parameter & $\begin{array}{l}\text { Healthy controls } \\
\qquad n=28\end{array}$ & $\begin{array}{c}\text { Diabetic patients } \\
\qquad n=51\end{array}$ & $P$-value \\
\hline \multirow[t]{5}{*}{$\mathrm{tcPO}_{2-}$ base $[\mathrm{mm} \mathrm{Hg}]$} & $56.3(18.6-81.1)$ & $52.8(27.9-80.8)$ & 0.22 \\
\hline & After controlling for: & Total cholesterol & 0.34 \\
\hline & & Triglycerides & 0.23 \\
\hline & & TSH & 0.40 \\
\hline & & fT4 & 0.19 \\
\hline \multirow[t]{5}{*}{$\mathrm{tcPO}_{2-}$ zero $[\mathrm{mm} \mathrm{Hg}]$} & $1.6(0.4-12.0)$ & $2.4(0.7-18.8)$ & 0.002 \\
\hline & Afte & ling for: Total cholesterol & 0.21 \\
\hline & & Triglycerides & 0.06 \\
\hline & & TSH & 0.09 \\
\hline & & fT4 & 0.054 \\
\hline \multirow[t]{5}{*}{$\operatorname{TTR}[\mathrm{s}]$} & $85(41-240)$ & $87(42-240)$ & 0.77 \\
\hline & Afte & ling for: Total cholesterol & 0.94 \\
\hline & & Triglycerides & 0.99 \\
\hline & & TSH & 0.63 \\
\hline & & fT4 & 0.99 \\
\hline \multirow[t]{5}{*}{ Slope } & $0.6(0.1-1.3)$ & $0.6(0.1-1.1)$ & 0.32 \\
\hline & Afte & ling for: Total cholesterol & 0.39 \\
\hline & & Triglycerides & 0.26 \\
\hline & & TSH & 0.50 \\
\hline & & fT4 & 0.21 \\
\hline
\end{tabular}

Values are presented as median and range.

Table 3. Multivariate analysis showing biochemical tcPO_z zero confounders

\begin{tabular}{|c|c|c|c|c|}
\hline \multirow[t]{2}{*}{ Parameter } & \multicolumn{2}{|c|}{$\begin{array}{l}\text { Healthy controls } \\
\qquad n=28\end{array}$} & \multicolumn{2}{|c|}{$\begin{array}{l}\text { Diabetic patients } \\
\qquad n=51\end{array}$} \\
\hline & $\beta$ & $P$-value & $\beta$ & $P$-value \\
\hline TSH & -0.13 & 0.52 & 0.01 & 0.99 \\
\hline fT4 & 0.14 & 0.53 & 0.14 & 0.30 \\
\hline Total cholesterol & -0.09 & 0.66 & 0.35 & 0.04 \\
\hline Triglycerides & -0.15 & 0.49 & -0.29 & 0.08 \\
\hline
\end{tabular}

diabetes than in healthy controls. No other differences between the groups were found (Table 1). There was no significant impact of gender on the tc $\mathrm{PO}_{2}$ parameters.

After adjusting for total cholesterol and triglycerides levels, the differences in $\mathrm{tcPO}_{2}$ between type 1 diabetic group and controls were no longer observed (Table 2). An analysis of the collected data showed no correlation between the age at type 1 diabetes onset, duration of the disease, insulin dose, treatment with pump, $\mathrm{HbA}_{1 c}$ level and $\mathrm{tcPO}_{2}$ parameters in the diabetic group. There were no correlations between serum concentrations of total cholesterol, triglycerides, TSH and ft4. Multivariate analysis showed an impact of total cholesterol on $\mathrm{tCPO}_{2-}$ zero in DM1 patients, but not in healthy controls (Table 3).

\section{Discussion}

In this study, we have observed an impairment in skin oxygenation reflected by a significant difference in the measured $\mathrm{tcPO}_{2}$ parameters between the diabetic and control groups. Adjustments for total cholesterol and triglycerides have eliminated the differences in the skin oxygenation between the groups. To the best of our knowledge, the effects of lipids on transcutaneous oxygen pressure have not been previously described in the literature. Due to the fact that lipids have an effect on endothelial function, thus impacting microcirculation [26-28], we assumed that they may influence $\mathrm{tcPO}$, as well.

Hypercholesterolemia is associated with significant retinal microvascular dysfunction as evidenced by a re- 
duction in flicker-induced dilatation of retinal arterioles [29]. Elevated low-density lipoprotein (LDL) cholesterol and triglycerides, lower high-density lipoprotein (HDL) cholesterol as well as older age, duration of diabetes, smoking, increased diastolic blood pressure and obesity, are all risk factors for peripheral neuropathy in type 1 diabetes [30]. Among adolescents with type 1 diabetes, the use of a statin did not change the albumin-to-creatinine ratio over time although it resulted in significant reductions in total, low-density lipoprotein and in triglyceride levels [31]. Research data show that the impaired skin microcirculation response can be reversed after cholesterol-lowering therapy [32, 33]. It is worth noticing that in both studied groups several subjects had extremely low tcPO ${ }_{2-}$ base values as it was measured in the forearm. These values were even lower than the threshold established for diagnosis of critical limb ischemia. However, direct comparison of these values may be misleading because of different electrode placement.

Studies of some authors have shown that tcPO is reduced in diabetes. These results coincide with the cooccurrence of neuropathy and may precede its appearance. However, these reports come from the 1980 s and 1990s [34-37]. During that time, less effective prevention, treatment and care could have been responsible for the observed changes in microcirculation even after a short duration of diabetes. Ewald's study shows an abnormal hyperaemic response in 13 diabetic children [34]. Skin postocclusive hyperaemia was recorded with a transcutaneous oxygen electrode at $37^{\circ} \mathrm{C}$ on admission before introducing insulin treatment and 1, 6, 12 and 24 months after diagnosis. To achieve congestion they used different temperatures.

It appeared that after diabetes has been present for 2 years, the vascular reactivity was significantly lower than in the control group. Breuer et al. designed a study in which they have examined 76 type 1 diabetic patients with disease duration ranging from several weeks to 30 years (median: 6.0 years) and mean $\mathrm{HbA}_{1 c}$ of $8.9 \%$. They showed reduced transcutaneous oxygen pressure associated with type 1 diabetes mellitus. This relationship was observed even in patients who at the time of the study showed no signs of micro- or macroangiopathy. The dependence of this phenomenon on the duration of diabetes was not revealed [35]. De Meijer et al. [23] reported that 60 adult type 1 and type 2 diabetic patients without signs of neuropathy had significantly decreased tcPO $\mathrm{P}_{2}$ values. They suggested $\mathrm{tc} \mathrm{PO}_{2}$ measurements in diabetic patients could be useful to detect subclinical microvascular impairment. In this study, there was no difference between the type 1 diabetes and control groups. When type 1 and type 2 diabetic patients were combined into one group, significant differences were found between the tcPO, values in the diabetic and nondiabetic groups.
Our study analysis of the influence of gender on tcPO 2 parameters did not show any differences either in the diabetes or the control group. Similar results were also obtained by Rodrigues who compared tcPO, between groups of adult men and women [37]. Conversely, a study by Orenstein et al. on healthy volunteers showed that women had had significantly higher $\mathrm{tc} \mathrm{PO}_{2}$ than men [38]. In Lagerkvist's study, the tcPO $\mathrm{Palue}_{2}$ obtained in healthy children and adolescents was 81 (65-97) $\mathrm{mm} \mathrm{Hg}$ [39], while in our study the $\mathrm{tcPO}$, value recorded on the arms of control subjects at baseline was 56.3 (18.6-81.1) $\mathrm{mm} \mathrm{Hg}$. The difference between the obtained results can be explained by different electrode placement and higher temperature used in Lagerkvist's study. In their study, patients were examined in a sitting position, with their left elbow joint in $90^{\circ}$ flexion and with their forearm resting horizontally on a pillow placed on their knees.

Most of the studies available in the literature concern adult patients with type 2 diabetes. Our study was specifically designed to be performed on children with type 1 diabetes with no clinically apparent microangiopathic complications in order to find other evidence of early deterioration of microcirculation. In the group of patients with diabetes, $\mathrm{tcPO}_{2}$ zero was significantly higher. Lack of data in the literature makes it impossible to refer to this parameter. It appears that this parameter indicates the efficiency of oxygen perfusion through tissues, i.e. skin flow. A higher tcPO, zero value indicates poorer skin microcirculation efficiency. The high-cholesterol levels adversely affect microvasculature. Hypercholesterolemia impairs endothelium dependent vasodilation because of defects in nitric oxide bioavailability. The pathogenic mechanism underlying microvascular dysfunction involves many steps that consequently lead to induction of inflammation and a prothrombotic phenotype [40]. After adjusting for in total cholesterol and triglyceride levels, skin flow disorders have been modified. Hence, it appears that the use of pharmacological intervention at an early stage of lipid disorders is able to reverse early impairments in skin microcirculation.

The limitation of our study may be the imbalance between the subgroup numbers, which could potentially influence the power of the study. Additionally, discussion of the results we have obtained is difficult due to the lack of other reports on patients with short-standing diabetes tested in a similar manner. However, our work brings a lot of new data into the discussion on transcutaneous oxygen pressure measurement as it was carried out on a large group of 79 children. So far, the studies with the highest numbers of participants have examined 158 individuals, only 76 of which were diabetic patients [35]. The projects in which children were studied were even less numerous [39].

The natural conclusion from the results obtained is the fact that it would be extremely important to analyse the parameters of transcutaneous oxygen pressure after 
treatment with statins due to reports of the protective nature of statins in other vascular areas [32, 33]. It seems that such a study could confirm our theoretical considerations in practice. Finally, we conclude that increased lipid levels are responsible for impairment of microvascular skin response to ischemic stimuli in short-lasting type 1 diabetes. Lipid-lowering therapy in children with type 1 diabetes, even in the absence of clinically evident microangiopathy, may be considered beneficial for skin microcirculation.

\section{Acknowledgments}

Jolanta Neubauer-Geryk and Melanie Wielicka both authors had an equal authorship in the study.

This study was supported by the State Committee for Scientific Research ST-120 and ST 02-0559/07/136 (Medical University of Gdansk, Poland). The funders had no role in study design, data collection and analysis, decision to publish, or preparation of the manuscript.

\section{Conflict of interest}

The authors declare no conflict of interest.

\section{References}

1. Kuryliszyn-Moskal A, Zarzycki W, Dubicki A, et al. A study on microvascular abnormalities in capillaroscopy in patients with type 1 diabetes mellitus. Diabetol Doświadcz Klin 2006; 6: 98-103.

2. Kuryliszyn-Moskal A, Dubicki A, Zarzycki W, et al. Microvascular abnormalities in capillaroscopy correlate with higher serum IL-18 and sE-selectin levels in patients with type 1 diabetes complicated by microangiopathy. Folia Histochem Cytobiol 2011; 49: 104-10.

3. Hosking SPM, Bhatia R, Crock PA, et al. Non-invasive detection of microvascular changes in a paediatric and adoles cent population with type 1 diabetes: a pilot cross-sectional study. BMC Endocr Disord 2013; 5: 41.

4. Jörneskog G, Brismar K, Fagrell B. Skin capillary circulation severely impaired in toes of patients with IDD with and without late diabetic complications. Diabetologia 1995; 38: 474-80.

5. Tibiriça E, Rodrigues E, Cobas RA, Gomes MB. Endothelial function in patients with type 1 diabetes evaluated by skin capillary recruitment. Microvasc Res 2007; 73: 107-12.

6. Jörneskog G, Brismar K, Fagrell B. Pronounced skin capillary ischemia in the feet of diabetic patients with bad metabolic control. Diabetologia 1998; 41: 410-5.

7. Moens AL, Goovaerts I, Claeys MJ, Vrints CJ. Flow-mediated vasodilation: a diagnostic instrument, or an experimental tool? Chest 2005; 127: 2254-63.

8. Serne EH, Gans RO, ter Maaten JC, et al. Capillary recruitment is impaired in essential hypertension and relates to insulin's metabolic and vascular actions. Cardiovasc Res 2001; 49: 161-8.

9. Serne EH, Stehouwer CD, ter Maaten JC, et al. Microvascular function relates to insulin sensitivity and blood pressure in normal subjects. Circulation 1999; 99: 896-902.
10. Ladurner R, Küper M, Königsrainer I, et al. Predictive value of routine transcutaneous tissue oxygen tension (tcPO2) measurement for the risk of non-healing and amputation in diabetic foot ulcer patients with non-palpable pedal pulses. Med Sci Monit 2010; 16: CR273-7.

11. Koch C, Chauve E, Chaudru S, et al. Exercise transcutaneous oxygen pressure measurement has good sensitivity and specificity to detect lower extremity arterial stenosis assessed by computed tomography angiography. Medicine 2016; 95: e4522.

12. Fife CE, Buyukcakir C, Otto GH, et al. The predictive value of transcutaneous oxygen tension measurement in diabetic lower extremity ulcers treated with hyperbaric oxygen therapy: a retrospective analysis of 1,144 patients. Wound Repair Regen 2002; 10: 198-207.

13. Moosa HH, Peitzman AB, Makaroun MS, et al. Transcutaneous oxygen measurements in lower extremity ischemia: effects of position, oxygen inhalation, and arterial reconstruction. Surgery 1988; 103: 193-8.

14. Nishio H, Minakata K, Kawaguchi A, et al. Transcutaneous oxygen pressure as a surrogate index of lower limb amputation. Int Angiol 2016; 35: 565-72.

15. Papa G, Spazzapan L, Pangos M, et al. Compared to coverage by STSG grafts only reconstruction by the dermal substitute Integra ${ }^{\circledR}$ plus STSG increases TCPO2 values in diabetic feet at 3 and 6 months after reconstruction. G Chir 2014; 35: 141-5.

16. Fife CE, Smart DR, Sheffield PJ, et al. Transcutaneous oximetry in clinical practice: consensus statements from an expert panel based on evidence. Undersea Hyperb Med 2009; 36: 43-53.

17. Fagher K, Katzman P, Löndahl M. Transcutaneous oxygen pressure as a predictor for short-term survival in patients with type 2 diabetes and foot ulcers: a comparison with ankle-brachial index and toe blood pressure. Acta Diabetol 2018; 55: 781-8.

18. American Diabetes Association. Microvascular complications and foot care, Sec. 9. In Standards of Medical Care in Diabetes. Diabetes Care 2016; 39 (Suppl. 1).

19. Singer DE, Nathan DM, Fogel HA, Schachat AP. Screening guidelines for diabetic retinopathy. American College of Physicians. American Diabetes Association and American Academy of Ophthalmology. Ann Intern Med 1992; 116: 683-5.

20. Dyck PJ. Detection, characterization, and staging of polyneuropathy: assessed in diabetics. Muscle Nerve 1988; 11: 21-32.

21. Lam YZ, Atkinson JK. Biomedical sensor using thick film technology for transcutaneous oxygen measurement. Med Eng Phys 2007; 29: 291-7.

22. Deng W, Dong X, Zhang Y, et al. Transcutaneous oxygen pressure (TCPO2): a novel diagnostic tool for peripheral neuropathy in type 2 diabetes patients. Diabetes Res Clin Pract 2014; 5: 336-43.

23. de Meijer VE, Van't Sant HP, Spronk S, et al. Reference value of transcutaneous oxygen measurement in diabetic patients compared with nondiabetic patients. J Vasc Surg 2008, 48: 382-8.

24. von Wirth F, Thomsen A, Jacobsen JB. The tcpO2 handbook. Radiometer Medical ApS, 2008.

25. Kernick DP, Tooke JE, Shore AC. The biological zero signal in laser Doppler fluximetry - origins and practical implications. Pflugers Arch 1999; 437: 624-31.

26. IJzerman RG, de Jongh RT, Beijk MA, et al. Individuals at increased coronary heart disease risk are characterized by an 
impaired microvascular function in skin. Eur J Clin Invest 2003; 33: 536-42.

27. Padró T, Vilahur G, Badimon L. Dyslipidemias and microcirculation. Curr Pharm Des 2018; 24: 2921-6.

28. Binggeli C, Spieker LE, Corti R, et al. Statins enhance postischemic hyperemia in the skin circulation of hypercholesterolemic patients: a monitoring test of endothelial dysfunction for clinical practice? J Am Coll Cardiol 2003; 42: 71-7.

29. Nägele MP, Barthelmes J, Ludovici V, et al. Retinal microvascular dysfunction in hypercholesterolemia. J Clin Lipidol 2018; 12: 1523-31.

30. Jaiswal M, Divers J, Dabelea D, et al. prevalence of and risk factors for diabetic peripheral neuropathy in youth with type 1 and type 2 diabetes: SEARCH for Diabetes in Youth Study. Diabetes Care 2017; 40: 1226-32.

31. Marcovecchio ML, Chiesa ST, Bond S, et al. ACE inhibitors and statins in adolescents with type 1 diabetes. N Engl I Med 2017; 377: 1733-45.

32. Rauch U, Osende JI, Chesebro JH, et al. Statins and cardiovascular diseases: the multiple effects of lipid lowering therapy by statins. Atherosclerosis 2000; 153: 181-9.

33. Haak E, Abletshauser C, Weber S, et al. Fluvastatin therapy improves microcirculation in patients with hyperlipidaemia. Atherosclerosis 2001; 155: 395-401.

34. Ewald U, Tuvemo T, Rooth G. Early reduction of vascular reactivity in diabetic children detected by transcutaneous oxygen electrode. Lancet 1981; 1: 1287-8.

35. Breuer HW, Breuer J, Berger M. Transcutaneous oxygen pressure measurements in type I diabetic patients for early detection of functional diabetic microangiopathy. Eur J Clin Investig 1988; 18: 454-9.

36. Railton R, Newman P, Hislop J, Harrower AD. Reduced transcutaneous oxygen tension and impaired vascular response in type 1 (insulin-dependent) diabetes. Diabetologia 1983; 25: 340-2.

37. Rodrigues $L M$, Pinto PC, Leal A. Transcutaneous flow related variables measured in vivo: the effects of gender. BMC Dermatol 2001; 1: 4.

38. Orenstein A, Mazkereth R, Tsur H. Mapping of the human body skin with the transcutaneous oxygen pressure method. Ann Plast Surg 1988; 20: 419-25.

39. Lagerkvist AL, Sten G, Redfors S, Holmgren D. Repeated blood gas monitoring in healthy children and adolescents by the transcutaneous route. Pediatr Pulmonol 2003; 35: 274-9.

40. Padró T, Vilahur G, Badimon L. Dyslipidemias and microcirculation. Curr Pharm Des 2018; 24: 2921-6. 